\title{
Measuring intake in free-living human subjects: a question of bias
}

\author{
Lauren Lissner $^{1 *}$, Berit L. Heitmann ${ }^{2}$ and Anna Karin Lindroos ${ }^{1}$ \\ ${ }^{1}$ Department of Internal Medicine, Göteborg University, 41345 Göteborg, Sweden \\ ${ }^{2}$ Danish Epidemiology Science Center, Institute of Preventive Medicine, Copenhagen, Denmark
}

Experimental as well as epidemiological nutrition studies often aim at measuring food intake and its correlates in 'free-living' human subjects, and the identification of optimal ways to do this is a challenge in both types of research. The purpose of the present paper is, first, to give a brief overview of general problems encountered in measuring intake in free-living human subjects. Focus will then be placed on issues involving assessment of nutritional exposures in observational epidemiology, a field that is vulnerable to many sources of measurement error. Finally, obesity will be presented as an example of a condition that can introduce bias in population-based surveys, as well as in clinical studies describing the obese condition.

\section{General considerations in observational studies}

Measuring usual dietary intake in free-living human subjects, besides being difficult to accomplish with precision, can in some instances represent a contradiction of terms. For instance, in quasi-experimental studies that provide controlled diets to subjects living, but not eating, in their natural environments, the generalizability of any findings to the subjects' own diets may be limited. Another problem with this type of study is that strict compliance to experimental regimens often poses a major problem, e.g. when subjects are living in their usual environments and have access to other foods. Finally, in the special case of ad libitum intake studies, while food intake can be measured easily and precisely in controlled situations, the quantity and quality of the food selected is likely to be altered as a consequence of the controlled condition itself.

In order to study eating behaviour under conditions of free consumption of usual diets, other approaches must be chosen. A few examples are: use of self-reported or proxyreported intakes; direct observation of subjects consuming their habitual diets in their natural environments; use of biomarkers for intake. Self- or proxy-reported intakes are often inaccurate and can introduce bias. Direct observation of subjects consuming their usual diets can be meaningful, but may affect behaviour unless done covertly, which is not always logistically or ethically feasible. Some biomarkers of dietary intake may be poorly correlated with the dietary indicator of interest (e.g. serum markers of vitamin status); others are expensive or invasive and thus impractical for large surveys (e.g. doublylabelled water, adipose tissue biopsies). The focus of the remaining presentation will be a discussion of issues and limitations of self-reported intake, the most common approach for assessing exposure in nutritional epidemiology.

\section{Measurement issues in nutritional epidemiology}

The measurement of usual food intake in large, free-living populations is a critical component of both descriptive and analytical nutritional epidemiology. However, as mentioned previously, methodological issues complicate the design and interpretation of these studies. Given the error with which diet is inevitably measured, together with the fact that dietary influences on health are often relatively small in magnitude, the statistical consequence is that large sample sizes are required. Thus, in the trade-off between using the most precise method $v$. assessing the diets of many individuals, sample size considerations often have priority.

Another general dilemma in nutritional epidemiology involves the fact that subjects who are asked to recall their recent intakes are limited by memory, subjects who are asked to record their intake as they consume it are likely to change their intake, and that subjects who are asked to report their usual intake are often unable to generalize with any accuracy. Furthermore, all three methods are potentially affected by various social influences dictating the type of diet that 'should' be reported, which can produce biased dietary information.

\section{Bias}

Rather than describing some of the available dietary methods at this point, it may be useful to focus on the issue of bias, a constant preoccupation among nutritional epidemiologists. Bias is defined as 'any process at any stage of inference which tends to produce results or conclusions that differ systematically from the truth' (Sackett, 1979). Biases can occur at any stage of research, from planning the

Abbreviations: SOS, Swedish Obese Subjects Intervention Study.

*Corresponding author: Lauren Lissner, fax +46 31 418527, email lauren.lissner@medfak.gu.se 
Table 1. Selected examples of bias that may apply to assessments of dietary exposure (From a more extensive list presented by Sackett, 1979)

\begin{tabular}{|c|c|c|}
\hline Type & Description & Comment \\
\hline Insensitive-measure bias & $\begin{array}{l}\text { When outcome measures are incapable of } \\
\text { detecting clinically significant associations }\end{array}$ & $\begin{array}{l}\text { May reflect difficulties in accurate recall, portion } \\
\text { estimation, and generalizing to 'usual diet' }\end{array}$ \\
\hline Underlying-cause bias (recall bias) & $\begin{array}{l}\text { Cases may ruminate about possible causes for } \\
\text { their illnesses and thus exhibit different recall } \\
\text { to previous exposures than controls }\end{array}$ & $\begin{array}{l}\text { Important to consider in case-control studies } \\
\text { involving dietary exposure with possible nutri- } \\
\text { tional cause }\end{array}$ \\
\hline Unacceptability bias & $\begin{array}{l}\text { Measurements which embarrass or invade } \\
\text { privacy may be systematically refused or } \\
\text { evaded }\end{array}$ & $\begin{array}{l}\text { Obese subjects may be particularly prone to this } \\
\text { in dietary surveys }\end{array}$ \\
\hline Obsequiousness bias & $\begin{array}{l}\text { Subjects may systematically alter responses in } \\
\text { the direction they perceive desired by the } \\
\text { investigator }\end{array}$ & $\begin{array}{l}\text { Especially relevant in face-to-face dietary inter- } \\
\text { view situations }\end{array}$ \\
\hline Expectation bias & $\begin{array}{l}\text { Observers may systematically err in measuring } \\
\text { and recording observations so that they } \\
\text { concur with previous expectations }\end{array}$ & $\begin{array}{l}\text { May occur in interview situation when unusual } \\
\text { diet is reported }\end{array}$ \\
\hline Exposure-suspicion bias & $\begin{array}{l}\text { A knowledge of the subject's disease may } \\
\text { influence both the intensity and outcome of a } \\
\text { search for exposure to the putative cause }\end{array}$ & $\begin{array}{l}\text { Possible in case-control studies where inter- } \\
\text { viewer is not blinded }\end{array}$ \\
\hline Attention bias & $\begin{array}{l}\text { Subjects may systematically alter their beha- } \\
\text { viour when they know they are being } \\
\text { observed }\end{array}$ & $\begin{array}{l}\text { Very important when the food-record method is } \\
\text { used }\end{array}$ \\
\hline
\end{tabular}

study to publishing the results. For the purpose of this discussion on reported food intake, many biases can occur when measuring dietary exposures; many of these were catalogued by Sackett (1979) and are listed in Table 1. Some of these biases (e.g. unacceptability bias, obsequiousness bias, expectation bias...) are normative, relating to perceptions of the acceptable or correct answer, and are particularly relevant when considering dietary exposures. Another type of bias, selection bias, often occurs in the recruitment stages, poses problems in generalizability of results, and is likely to occur in all types of studies where participation is incomplete. Selection bias is of particular concern in descriptive nutritional surveys, since there is reason to believe that non-responders have different, probably less healthy, dietary habits than participants. It is important to remember that the various biases are not mutually exclusive, and may be operating simultaneously in a single dietary survey. Furthermore, while some of these biases may be reduced, for instance by using repeat measurements or 'blinding' the observers, some of the normative biases may be impossible to eliminate.

One key issue in epidemiology when considering bias is whether the dietary exposure error is non-differential or differential. Non-differential misclassification affects different disease-outcome groups similarly, whereas a differential error affects the main study groups in different ways. It was previously assumed that non-differential errors result in conservative estimates of effects, although exceptions have been shown (Dosemeci et al. 1990; Flegal et al. 1991). In contrast, when the dietary misclassification has a different distribution in subjects with and without the disease of interest, it should be clear that erroneous conclusions can be drawn. A frequently described scenario for this type of differential bias is the case-control study, in which diet is assessed retrospectively when the cases (and/or interviewers) have knowledge of the subject's disease status. This opens the possibility for differential recall bias in which subjects with a disease recall their diet with different accuracy compared with subjects without the disease.

Possible consequences of this type of bias were presented in a study of the association between diet and breast cancer, using dietary assessments conducted both before and after diagnosis (Giovannucci et al. 1993). Specifically, a case-control type analysis of earlier diet, as recalled by cases after diagnosis, revealed an elevated odds ratio suggesting excess risk in association with high fat intake. In contrast, a nested case-control analysis, based on data collected before diagnosis, revealed no excess risk in association with fat intake. This was interpreted as evidence of a differential bias, most probably a recall bias, derived from knowledge of the disease and its possible underlying nutritional cause.

\section{Choice of dietary methods}

In the context of these various biases, it is informative to discuss some of the different methods available to nutritional epidemiologists. The diet-record method was traditionally considered the most accurate food intake method, but like most methods, validity depends on subjects' willingness to cooperate. Because of problems related to compliance, requesting more than seven consecutive days of record keeping is not suggested (Bingham, 1987), although considerably longer recording periods may be required to capture usual intakes of a number of nutrients (Nelson et al. 1989). Subjects in one study involving food records reported that a regimen of weighing all food before consumption had interfered with normal eating behaviour, which affected their motivation to continue the study (Livingstone et al. 1990). Recently, other investigators found that almost half the subjects completing weighed-food records admitted altering their intake in some way, due to inconvenience, being selfconscious, or being ashamed (Macdiarmid \& Blundell, 1997). 
Alternative diet-reporting methods have other limitations. The $24 \mathrm{~h}$ method, on average, underestimates food intake (Bingham, 1987) due for instance to limits of memory, selective omissions, insufficient interviewer probing. A traditional diet history is labour intensive and cannot be self-administered, although the data quality may be higher, e.g. with respect to total energy estimation (Black et al. 1991a). A food-frequency questionnaire can be very convenient to analyse when optically scanned, but it is inflexible with regard to unusual food intake patterns, and becomes obsolete when used longitudinally under conditions of a changing product availability, e.g. introduction of fat-reduced items. Furthermore, it has been pointed out that grouping of foods in a frequency questionnaire can result in substantial misclassification (Sempos, 1992). For instance, combining all dessert pies into one response category would produce error if vitamin A were the nutritional exposure of interest, given that apple and pumpkin pies contain $3 \nu$. $240 \mu \mathrm{g}$ respectively.

\section{Validation studies in nutritional epidemiology}

In view of the wide variety of biases that may affect the quality of data in nutritional epidemiology, it is necessary to consider validation as early as possible in the course of the study. Specifically, validity refers to the degree to which a dietary measurement method actually measures the aspect of the diet that it was designed to measure (Willett, 1990). In assessing validity, it should be kept in mind that an unbiased 'gold standard' is often not available for assessing instrumentation. Thus, the concept of 'relative validity' has also been adopted, e.g. when comparing a method designed for rapid data handling with a labourintensive method, such as multiple food records, which are assumed to be more accurate.

An important aspect of validation is the question of whether error is random or systematic. When describing the intake levels of populations, it is important to have a method that gives a correct group mean, and random error will not affect this value in large studies. Thus, in studies of nutritional status of a population in relation to dietary recommendations, it may be particularly relevant to study absolute agreement with the best available standard. In contrast, in analytical epidemiology, we are interested in ranking individuals with respect to dietary exposure and correlating this value with an outcome. In this case, it may be more important to have a correct rank than a correct group mean. A theoretical example of this would be total fat intake; if all individuals under-reported their intake to a similar extent with a systematically-biased method, this would have no bearing on the presence or strength of the association. However, it may be counter-argued that even if ranking preserves the correct risk ratio under conditions of systematic error, incorrect absolute levels make it difficult to formulate public health recommendations concerning the exposure level at which health risk increases.

\section{Validation of total energy}

The current 'gold standard' for evaluating energy intakes in free-living subjects is by means of doubly-labelled water, and the feasibility of using this method for validating selfreported intakes is increasing. One less-expensive method for validating reported dietary intakes in general, and energy in particular, has involved the so-called physical activity level values (Goldberg et al. 1991). This method typically estimates resting metabolic rate from prediction equations based on various combinations of sex, weight, height, and age; alternatively, measures of body fat and fatfree mass may be used. The ratio, reported intake:predicted resting expenditure will vary as a function of physical activity level. Individuals with reported intakes that are less than a certain proportion of predicted resting expenditures are assumed by this approach to be giving invalid data, and are usually recommended for exclusion from further study. In general, the high end of the physical activity level distribution, being less easily defined, is not subjected to any such cut-off, and hence probably overreporting is not considered.

Using this approach, a review including thirty-seven published dietary studies illustrated that there is a substantial difference in the validity of dietary assessment methods. Compared with habitual energy expenditure, estimated from height and weight, $88 \%$ of the diet recalls, $64 \%$ of the diet records and $25 \%$ of the diet histories included in the review presented energy intakes that were below those needed for maintaining a sedentary lifestyle (Black et al. 1991a). Using this method to identify individuals who are under-reporting is not completely straightforward, due to the assumptions of the method. For instance, sedentary individuals are more likely to be excluded than physically-active individuals, given the same degree of under-reporting. Thus, it is important to validate energy intakes with objective methods such as doublylabelled water, when possible.

\section{Validation of protein}

A well-known biomarker for external evaluation of the validity of habitual food intake is estimation of protein intake from $24 \mathrm{~h}$ urinary $\mathrm{N}$ output (Isaksson, 1980; Bingham \& Cummings, 1985). In order to monitor completeness, subjects can be given tablets containing $p$ aminobenzoic acid to be taken during the day of urine collection. Simultaneous use of both $24 \mathrm{~h}$ urinary $\mathrm{N}$ and doubly-labelled water has indicated that the methods identify the same subjects as reporting incorrect food intakes (Black et al. 1991b).

\section{Validation of macronutrient-energy density}

A number of studies have compared nutrient densities by two different methods and arrived at the conclusion that different methods capture different macronutrient profiles (Flegal \& Larkin, 1990; Rutishauser, 1995). Other studies have shown similar macronutrient-energy densities as reported by different methods, even when absolute energy intakes differ, suggesting that individuals report the same types of diets with different methods (Lissner \& Lindroos, 1994). Those studies finding similar nutrient profiles are limited by the possibility that the same types of selfreporting biases are inherent in the different methods, while 
in studies finding different nutrient profiles it is not clear which of the two methods is closer to the true diet. However, results from both types of study are limited by the fact that no dietary-reporting method can be assumed to be unbiased. Hence, the question of whether errors in food intake are macronutrient-specific can only be addressed by means of applying an external validation.

One approach for doing this may be referred to as protein-energy density validation (Heitmann \& Lissner, 1995). By estimating the degree of reporting error of energy from protein relative to total energy, bias in protein density of the diet may be estimated. The degree of underreporting of protein is obtained by comparing protein intake data with estimates from $24 \mathrm{~h}$ urinary $\mathrm{N}$ excretion. The degree of under-reporting of energy is obtained by comparing energy intake with estimates of $24 \mathrm{~h}$ energy expenditure, the latter using impedance-based body composition and self-reports of physical activity. Proportional over-reporting of protein would imply proportional underreporting of the other macronutrient fractions. The validity of this method for assessing under-reporting in individuals is dependent on the quality of the energy expenditure method, and specifically on the assumption that adjustments for reported physical activity are unbiased.

A complete discussion of other biomarkers that may be used in validation studies is beyond the scope of the present paper. Examples of biochemical indicators that are used in nutritional epidemiology, as well as in the specific context of validation, include adipose tissue biopsies to validate fatty acid intake; serum levels of reported micronutrients are discussed elsewhere (Hunter, 1990).

\section{Dietary instrumentation issues in relation to obesity}

Obesity represents a classic example of problems encountered when reported nutrient intakes are used. In the past, the view that obesity results from overeating was challenged by a number of studies in which obese persons did not report higher intakes than normal-weight subjects (Keen et al. 1979; Kromhout, 1983; Miller, 1991). However, with the introduction of the doubly-labelledwater technique it became evident that obese subjects expend more energy than normal-weight subjects and also tend to under-report energy intake to a greater extent than normal-weight subjects (Prentice et al. 1986; Bandini et al. 1990). The dietary-history method appears to give the best results in obese individuals, but this method is very timeconsuming and may be subject to interviewer bias (Beaudoin \& Mayer, 1953; Black et al. 1993). It is generally believed that obese subjects are particularly susceptible to a variety of normative biases. For instance, food record keeping may often be inappropriate for the obese, in view of the likelihood that obese subjects may have used the food-recording technique as a behavioural monitoring technique in previous weight-loss attempts. Furthermore, several days of recording may not capture binge-eating episodes that occur in some obese individuals, even if these episodes were to be accurately reported.

\section{Measuring intake in the obese: an example from the Swedish Obese Subjects Intervention Study}

The Swedish Obese Subjects Intervention Study (SOS; Sjöström et al. 1992) has developed a self-administered dietary assessment method that is specifically designed to capture dietary intakes of obese subjects. The method is based on a previously-validated method developed in Sweden, which was refined by incorporating additional questions describing problematic eating characteristics of obese individuals. The validity of the dietary questionnaire was then tested in relation to predicted energy expenditure (BMR, adjusted for physical activity level) and judged to be satisfactory in obese and non-obese subjects (Lindroos et al. 1993). To permit comparison of the SOS method with a traditional dietary method, each subject also completed a $4 \mathrm{~d}$ food record. In both the obese and the non-obese groups, the SOS questionnaire-based energy intake was $4 \%$ higher than estimated energy expenditure (not significant), with a between-method correlation of $0.34(P<0.01)$. In contrast, the $4 \mathrm{~d}$ food record underestimated energy expenditure by $23 \%$ in the obese subjects $(P<0.0001)$, but gave reasonable energy intake levels in the non-obese. The correlation between the SOS method and energy expenditure, although not as high as one would like, gives evidence that the method can rank obese and non-obese subjects. The question remains of whether any macronutrient distortion is occurring even in the context of valid energy intakes.

\section{Is obesity-related under-reporting occurring at the whole-diet level? (evidence for and against)}

If obese subjects in particular under-report specific components of their diet more than normal-weight subjects, this would have even more serious implications for the interpretation of dietary survey data than under-reporting at the whole-diet level. Inflation or deflation of diet-disease associations as a result of generalized under-reporting might be correctable to some extent by one of the energy adjustment procedures. In contrast, errors from nutrientspecific under-reporting could create a range of unpredictable biases.

Recent research seems to show that snacks are differentially under-reported relative to the rest of the diet, an observation which could be a cause for concern if obese individuals consumed more snacks. In one controlled study in which ad libitum food intake was covertly measured, food items consumed during meals were accurately reported, while snack foods eaten between meals were highly under-reported, a bias which was not, however, specific to obese subjects (Poppitt $e$ t al. 1995). This finding is consistent with comments from a study in which food intake was assessed by $7 \mathrm{~d}$ weighed record, where participants admitted that they had omitted or simplified some measurements because they found that having to weigh snacks was particularly onerous and irritating (Livingstone et al. 1990).

Using another method, obesity-related distortion of macronutrient distribution seemed to be occurring in obese subjects. Specifically, by using the validation method for 
protein-energy density, we have observed that lean subjects in general reported their food intake accurately, whereas obese subjects under-reported both protein and energy intake (Heitmann \& Lissner, 1995). However, among obese subjects, under-reporting was approximately $15 \%$ greater for energy than for protein, suggesting a differential underreporting pattern for the other macronutrient sources. Hence, the results of that study are in agreement with the general assumption that the obese tend to under-report lowprotein foods (e.g. fat- and/or carbohydrate-rich foods), rather than equally under-report food intake of all kinds.

A further example is from SOS, where the macronutrient-energy densities agreed closely when assessed by the $4 \mathrm{~d}$ record and the SOS method, indicating that a similar distribution of macronutrients was being given with the two reporting methods (Lissner \& Lindroos, 1994). However, further examination of the data using validation for proteinenergy density, matched earlier findings in the Danish sample (Heitmann \& Lissner, 1995), i.e. on a proportional basis the obese subjects over-reported their percentage of energy from protein, indicating that they were underreporting the percentage energy from fat plus carbohydrate sources. In contrast, no significant difference was seen in the non-obese group. Similar patterns were observed using energy residual analysis instead of percentage energy when contrasting protein values between methods. The results are shown in Table 2 and they suggest that agreement between two reporting methods may not guarantee that they are unbiased. While the SOS method has made it feasible to obtain valid estimates of total energy in obese and nonobese subjects, we cannot exclude the possibility that obesity-related biases in macronutrient distribution remain. However, possible differences in weight stability of the obese and non-obese groups during the observation period make it necessary to qualify this observation.

With methods that are presently available, it is not possible to further specify which non-protein component of the diet, i.e. fat, sugar, other carbohydrate sources, or alcohol, is being under-reported. Furthermore, the two examples of apparent macronutrient distortions by the obese reported previously may be contrasted with a smaller study of successful slimmers, presumably formerly obese, in which it was concluded that energy and protein were underestimated to a similar extent (Black et al. 1991b). This latter study is the only one that is based on doublylabelled water for energy determination. Thus, despite general agreement that most methods underestimate total intake in the obese, the question of whether selective underreporting occurs with obesity is not fully answered.

\section{Consequences of obesity-related under-reporting in epidemiology}

The problem with obtaining valid dietary information has implications for the interpretation of diet-disease relationships, since bias in diet reporting may explain a positive, a negative, or a null finding. This is particularly the case if the reporting bias is differential, e.g. applies differently to different disease groups being studied. The studies described earlier in the present paper raise the question of possible effects of obesity-related under-reporting on disease-related risk estimates.

One of the most controversial nutrients in epidemiology is dietary fat, and it is thus of interest to understand how obesity-related under-reporting of dietary fat could affect epidemiological associations. Whereas most experimental studies in this area show that a reduction in dietary fat is associated with a reduction in levels of certain cardiovascular risk factors, the evidence from prospective epidemiological studies is much weaker. It has been assumed that the weaker correlations seen in observational studies are dependent on poor quality of information on diet, and that true risk associated with high dietary fat intake is underestimated in these studies.

Table 2. Protein and total energy intake from the Swedish Obese Subjects Intervention Study dietary questionnaire compared with nitrogen excretion in urine and estimated $24 \mathrm{~h}$ energy expenditure (EE) (Mean values and standard deviations)

\begin{tabular}{|c|c|c|c|c|}
\hline & \multicolumn{2}{|c|}{ Non-obese (n 19) } & \multicolumn{2}{|c|}{ Obese ( $n 44)$} \\
\hline & Mean & SD & Mean & SD \\
\hline \multicolumn{5}{|l|}{ Protein intake $(\mathrm{g})$} \\
\hline Dietary questionnaire & $91 \cdot 2$ & 24.8 & 110.8 & $36 \cdot 1$ \\
\hline Based on urine & 91.5 & $20 \cdot 2$ & 95.9 & 21.3 \\
\hline Questionnaire - urine & -0.3 & $(\mathrm{NS})^{*}$ & 14.9 & $(P=0.011)^{*}$ \\
\hline \multicolumn{5}{|l|}{ Energy (MJ) } \\
\hline Intake questionnaire & 9.72 & 2.4 & 11.4 & 3.7 \\
\hline Estimated $24 \mathrm{EE}$ & 9.09 & 1.2 & 10.9 & 1.7 \\
\hline Questionnaire - $24 \mathrm{EE}$ & 0.6 & $(\mathrm{NS})^{*}$ & 0.5 & $(\mathrm{NS})^{*}$ \\
\hline \multicolumn{5}{|c|}{ Percentage energy from protein } \\
\hline Dietary questionnaire & $15 \cdot 8$ & 1.82 & $16 \cdot 4$ & 1.81 \\
\hline Based on urine & $16 \cdot 9$ & $3 \cdot 10$ & $15 \cdot 0$ & 3.46 \\
\hline Questionnaire - urine & $-1 \cdot 1$ & $(\mathrm{NS})^{\star}$ & 1.4 & $(P=0.011)^{*}$ \\
\hline \multicolumn{5}{|l|}{ Protein adjusted $^{\dagger}(\mathrm{g})$} \\
\hline Dietary questionnaire & 91.2 & $10 \cdot 3$ & 110.6 & $12 \cdot 0$ \\
\hline Based on urine & $91 . \overline{5}$ & 16.7 & 95.0 & 20.2 \\
\hline Questionnaire - urine & -0.3 & $(\mathrm{NS})^{\star}$ & 15.6 & $(P=0.0001)^{*}$ \\
\hline
\end{tabular}


(a) Overestimated association

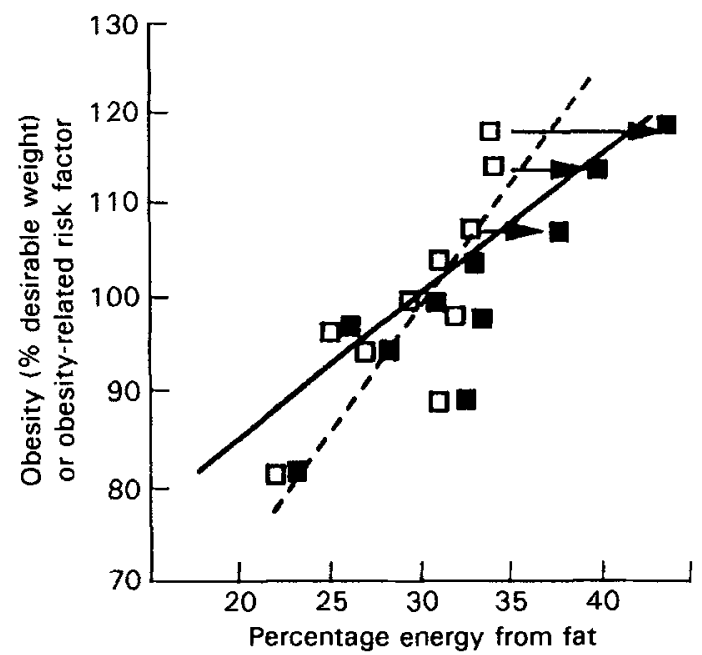

(b) Hidden association

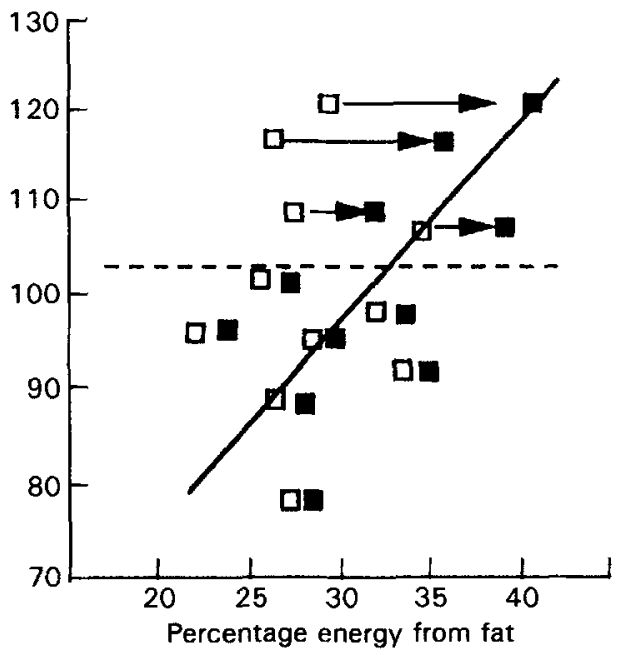

Fig. 1. Potential consequences of obesity-related under-reporting of dietary fat, using hypothetical data points. (- - ), Might be formed by reported values $(\square) ;(-)$, formed by true values $(\square) . \longrightarrow$, Hypothetical error due to obesity-related under-reporting.

Fig. 1 gives a theoretical example of potential consequences of obesity-related under-reporting of dietary fat. Let us start with a simple example of obesity as the pathology of interest. It seems obvious that under-reporting of dietary fat by obese individuals would obscure any positive association between dietary fat and obesity. This situation is illustrated in Fig. 1(b). It is possible to take this argument one step further and apply it to obesity-related health end-points. Specifically, if the individuals who are (or will be) cases are more likely than controls to be overweight and, thus, more likely to under-report their fat intake, associations may also be distorted. In contrast, Fig. 1(a) illustrates that bias may accentuate the apparent magnitude of the association. The situations described in Fig. 1 are analogous to positive and negative confounding, which can also bias an association in both directions. However, in contrast to the situation of confounding by obesity, in the present case obesity may act both as a source of biased measurement error and as a biological mediator of the health end-point. Thus, it seems safe to conclude that obesity has the potential for creating serious instabilities in observed diet-disease relationships.

There are examples in the literature of results that might be explained by obesity-related reporting bias. One example is the findings from the Chicago Western Electric Study, in which body fatness modified the association between dietary and serum cholesterol levels (Goff $e t$ al. 1993). Whether this finding can be explained by differential reporting accuracy or differential physiological responsiveness to dietary changes is not known. However, the authors pointed out that no such modification was seen for the relationship between saturated fatty acid intake and serum cholesterol, evidence which indirectly argues against an obesity-related artifact. Another example involves studies of temporal eating behaviours among obese $v$. non-obese subjects, which have raised the 'nibbler $v$. gorger' distinction in comparing eating patterns of non-obese $v$. obese subjects respectively (Fábry \& Tepperman, 1970). Again, if the obese differentially under-report their snack intake more than the non-obese, any associations between snacking patterns and obesity or its co-morbidities would be artifactual.

\section{Conclusion}

Measurement of dietary intake in free-living subjects is a major challenge in nutrition research. There are many potential types of bias to be considered, and some of these seem to be more prevalent when studying reported dietary intakes of obese subjects. Since many diseases are strongly obesity-related, a bias related to obesity could be translated into a disease-related bias. If it is true that the obese specifically under-report a dietary constituent (e.g. fat), this could result in a compound-misclassification bias, consisting of general plus macronutrient-specific under-reporting by obese subjects, who may also be more likely to be cases. This reporting bias could either attenuate or exaggerate associations with obesity-related disease. This problem has not been given sufficient attention in nutritional epidemiology. Efforts should continue with regard to developing improved methods that have comparable precision in normal-weight and overweight subjects. In circumstances where normative biases in reported diet are unavoidable, the statistical consequences of distorted nutrient profiles should be further explored, including situations in which the error is and is not differentially distributed by disease status.

\section{References}

Bandini LG, Schoeller DA \& Dietz W (1990) Energy expenditure in obese and nonobese adolescents. Pediatric Research 27, 198-202.

Beaudoin R \& Mayer J (1953) Food intakes of obese and nonobese women. Journal of the American Dietetic Association 29, 29-33.

Bingham SA (1987) The dietary assessment of individuals; methods, accuracy, new techniques and recommendations. Nutrition Abstracts and Reviews 57, 705-742. 
Bingham S \& Cummings $J$ (1985) Urine nitrogen as an independent validatory measure of dietary intake: a study of nitrogen balance in individuals consuming their normal diet. American Journal of Clinical Nutrition 42, 1276-1289.

Black A, Prentice A, Goldberg G, Jebb S, Bingham S, Livingstone B \& Coward A (1993) Measurements of total energy expenditure provide insights into the validity of dietary measurements of energy intake. Journal of the American Dietetic Association 93, 572-579.

Black AE, Goldberg GR, Jebb SA, Livingstone MBE, Cole TJ \& Prentice AM (1991a) Critical evaluation of energy intake data using fundamental principles of energy physiology: 2. Evaluating the results of published surveys. European Journal of Clinical Nutrition 45, 583-599.

Black AE, Jebb SA \& Bingham SA (1991b) Validation of energy and protein intakes assessed by diet history and weighed records against energy expenditure and $24 \mathrm{~h}$ urinary nitrogen excretion. Proceedings of the Nutrition Society 50, $108 \mathrm{~A}$.

Dosemeci M, Wacholder S \& Lubin JH (1990) A brief original contribution. Does nondifferential misclassification of exposure always bias a true effect toward the null value? American Journal of Epidemiology 132, 746-748.

Fábry P \& Tepperman J (1970) Meal frequency - a possible factor in human pathology. American Journal of Clinical Nutrition 23, 1059-1068.

Flegal KM, Keyl PM \& Nieto FJ (1991) Differential misclassification arising from nondifferential errors in exposure measurement. American Journal of Epidemiology 134, 12331244.

Flegal KM \& Larkin FA (1990) Partitioning macronutrient intake estimates from a food frequency questionnaire. American Journal of Epidemiology 131, 1046-1058.

Giovannucci E, Stampfer MJ, Colditz GA, Manson JE, Rosner BA, Longnecker M, Speizer FE \& Willett WC (1993) A comparison of prospective and retrospective assessments of diet in the study of breast cancer. American Journal of Epidemiology 137, 502-511.

Goff DC, Shekelle RB, Moyé LA, Katan MB, Gotto AM \& Stamler J (1993) Does body fatness modify the effect of dietary cholesterol on serum cholesterol? Results from the Chicago Western Electric Study. American Journal of Epidemiology 137, 171-177.

Goldberg GR, Black AE, Jebb SA, Cole TJ, Murgatroyd PR, Coward WA \& Prentice AM (1991) Critical evaluation of energy intake data using fundamental principles of energy physiology: 1. Derivation of cut-off limits to identify underrecording. European Journal of Clinical Nutrition 45, 569-581.

Heitmann B \& Lissner L (1995) Dietary under-reporting by obese individuals: Is it specific or nonspecific? British Medical Journal 311, 986-989.

Hunter D (1990) Biochemical indicators of dietary intake. In Nutritional Epidemiology, pp. 143-216 [W Willett, editor]. New York: Oxford University Press.
Isaksson B (1980) Urinary nitrogen output as a validity test in dietary surveys. American Journal of Clinical Nutrition 33, 4-5.

Keen H, Thomas BJ, Jarrett RH \& Fuller JH (1979) Nutrient intake, adiposity, and diabetes. British Medical Journal 1, 655-658.

Kromhout D (1983) Energy and macronutrient intake in lean and obese middle-aged men (the Zutphen Study). American Journal of Clinical Nutrition 37, 295-299.

Lindroos AK, Lissner L \& Sjöström L (1993) Validity and reproducibility of self-administered dietary questionnaire in obese and non-obese subjects. European Journal of Clinical Nutrition 47, 461-481.

Lissner L \& Lindroos AK (1994) Is dietary underreporting macronutrient-specific? European Journal of Clinical Nutrition 48, 453-454.

Livingstone MBE, Prentice AM, Strain JJ, Coward WA, Black AE, Barker ME \& McKenna PG (1990) Accuracy of weighed dietary records in studies of diet and health. British Medical Journal 300, 708-712.

Macdiarmid JI \& Blundell JE (1997) Short report. Dietary underreporting: what people say about recording their food intake. European Journal of Clinical Nutrition 51, 199-200.

Miller WC (1991) Diet composition, energy intake, and nutritional status in relation to obesity in men and women. Medicine and Science in Sports and Exercise 23, 280-284.

Nelson M, Black AE, Morris JA \& Cole TJ (1989) Between- and within-subject variation in nutrient intake from infancy to old age: estimating the number of days required to rank dietary intakes with desired precision. American Journal of Clinical Nutrition 50, 155-167.

Poppitt SD, Swann D, Black AE \& Prentice AM (1995) Is underreporting of energy intake in obese women macronutrient specific? Covert measurements in a metabolic facility. International Journal of Obesity 19, 29 Abstr.

Prentice AM, Black AE, Coward WA, Davies HL, Goldberg GR, Murgatroyd PR, Ashford J, Sawyer M \& Whitehead RG (1986) High levels of energy expenditure in obese women. British Medical Journal 292, 983-987.

Rutishauser I (1995) Is dietary underreporting macronutrient specific? European Journal of Clinical Nutrition 49, 219-220.

Sackett DL (1979) Bias in analytic research. Journal of Chronic Disease 32, 51-63.

Sempos CT (1992) Invited commentary: Some limitations of semiquantitative food frequency questionnaires. American Journal of Epidemiology 135, 1127-1132.

Sjöström L, Larsson B, Backman L, Bengtsson C, Bouchard C, Dahlgren S, Hallgren P, Jonsson E, Karlsson J, Lapidus L, Lindroos AK, Lindstedt S, Lissner L, Narbro K, Näslund I, Olbe L, Sullivan M, Sylvan A, Wedel H \& Ågren G (1992) Swedish obese subjects (SOS). Recruitment for an intervention study and a selected description of the obese state. International Journal of Obesity 16, 465-479.

Willett W (editor) (1990) Reproducibility and validity of foodfrequency questionnaires. In Nutritional Epidemiology, pp. 92126. New York: Oxford University Press. 